\title{
Astrocyte-like glia-specific gene deathstar is crucial for normal development, adult locomotion and lifespan of male Drosophila
}

Hadi Najafi

University of Ottawa

Kyle Wong

University of Ottawa

Woo Jae Kim ( $\square$ woo.jae.kim@uottawa.ca )

University of Ottawa

Research article

Keywords: Astrocyte-like glia, CG11000 gene, Cell type, Male development, Drosophila melanogaster, Single cell RNA-seq, Specific marker, deathstar

Posted Date: February 12th, 2020

DOI: https://doi.org/10.21203/rs.2.19590/v2

License: (c) (i) This work is licensed under a Creative Commons Attribution 4.0 International License. Read Full License 


\section{Abstract}

Background Drosophila melanogaster is a proper model organism for studying the development and function of the nervous system. The Drosophila nervous system consists of distinct cell types with significant homologies to various cell types of more advanced organisms, including human. Among all cell types of the nervous system, astrocyte-like glia (ALG) have conserved functions to mammals and are essential for normal physiology and behaviours of the fly.

Results In this study, we exploited the gene expression profile of single cells in Drosophila optic lobe to identify the genes with specific expression pattern in each cell type. Through a bioinformatical analysis of the data, a novel ALG-specific gene (here assigned as deathstar) was identified. Immunostaining of deathstar in the central nervous system (CNS) showed its presence in specific regions of Drosophila ventral nerve cord, which previously has been characterized as ALG cells. Consistent with the bioinformatical analysis, deathstar -related signals were overlapped with the signals of the previouslyreported ALG marker, Eaat1, supporting its specific expression in ALG cells. At the physiological level, RNAi-mediated suppression of deathstar gene impeded the normal development of male flies without any effects on females. Cell type-specific expression of deathstar RNAi showed that deathstar gene affects locomotion behaviour and lifespan of D. melanogaster, in an ALG-specific manner.

Conclusions Taken together, we showed that bioinformatical analysis of a previously reported expression data of Drosophila optic lobe successfully predicted the ALG-specific expression pattern of deathstar gene. Moreover, it was consistent with the ALG-specific effects of this gene on locomotion and lifespan of D. melanogaster, in vivo .

\section{Background}

The function of the central nervous system (CNS) depends on a complex network of several neuronal and glial cell types [1, 2]. Due to the feasibility of genetic manipulation and behavioral analysis, Drosophila melanogaster has emerged as a powerful model organism for studying the development and function of the nervous system $[3,4]$. There is a functional homology between the cell types in the nervous system of D. melanogaster and those of more advanced organisms, including human [5]. Glial cells constitute an essential part of CNS [5] , and until now, several distinct types of these cells were also identified in the Drosophila nervous system [6, 7]. Each cell types have a distinct profile of gene expression [8] and have their specific functions matched to their physical territories or physiological states [9]. Based on the differences in gene expression profile, each cell type of Drosophila nervous system can be distinguished by a set of genes, which are known as molecular markers for each cell type [6].

In addition to the existed molecular markers for each cell type, there may be some uncharacterized genes in D. melanogaster, which are capable of discerning a specific cell type in the nervous system. Moreover, a specific cell type likely differs only in the expression of the limited number of genes which confer a new function to it $[10,11]$. In this study, we used a publicly available expression profile of 55,000 single cells 
of Drosophila optic lobe in order to find novel molecular markers for specific cell types, following by experimental confirmation of the predicted results. Here, we studied the CG11000 gene which was previously reported as an uncharacterized protein-coding gene with 332 amino acids. It is located on the chromosome 3 on positive strand (3R:5,802,328..5,813,258 [+]) and reported as membrane protein with possible functional role in $D$. melanogaster development. To date, there is no functional study for this gene in D. melanogaster.

\section{Results}

\section{Cell type identification of the single cells of Drosophila optic lobe based on their specific markers}

To identify the cells corresponding to distinct subtypes of glia and neurons, the expression profile of the sequenced single cells by Konstantinides et al. [12] was analyzed based on their previously-reported marker genes (Fig. 1A). According to the expression profile of 55000 sequenced single cells, the cells with the highest expression of each cell type-specific marker were selected and classified. A total of forty-two cells were identified and classified into seven cell types. The heatmap of gene expression for these selected cells confirmed the unique expression pattern of each marker in the corresponding cell types (Fig. 1B). CG4797 and gem are differentially expressed in PNG, and SPG were distinguished by the three markers Mdr65, Moody and Gli.

Other glial cells were successfully distinguished by single marker;

Cyp4g15, CG9657, CG34340 and Eaat1. Moreover, these genes could individually separate CG, EGN, EGT and ALG, respectively. Neuronal cells were determined based on the highest expression of $n$ Syb and elav genes (Fig. 1B).

\section{Hierarchical clustering of Drosophila neural cell subtypes based on their similarities in transcriptome}

As shown above, different types of Drosophila neural cells could be classified based on some specific marker genes (Fig. 1). In order to know whether the classified cells in each category have the same expression profile or not, correlation analysis between the selected cells was carried out based on their transcriptome analysis. Results showed that most of the cells expressing the same genetic marker(s) tend to be clustered together, suggesting their similar transcriptome and accuracy of the identification of cell types (Fig. 2A). However, the cells with the highest expression of SPG markers and some other cells could not be classified in a single cluster suggesting their transcriptome dissimilarity or partial insufficiency of the available genetic markers for clustering (e.g. expression heterogeneity of the SPG markers: Mdr65, Moody and G/i).

With at least 3 of 6 similar cells in each category, differential expression analysis was performed in order to find the genes with specific expression in each cell type. While the identified PNG were positive for the expression of their two known genetic markers (CG4797 and gem) (Fig. 1B), there were two sub-clusters of PNG (PNG- $\alpha$ and PNG- $\beta$ ) in our transcriptome analysis which suggests the presence of a novel subtype for PNG (Fig. 2A). This analysis was also performed on another set of 42 cells of 55,000 cells and the 
same results were obtained (Supplemental file S1), which could support the accuracy of cell type characterization and clustering.

Also, the list of differentially expressed genes for each cell type in addition to differentially expressed genes between the two subtypes of PNG is presented in supplemental file S2.

Using the transcriptome data of the studied cell types and their clustering results, we assigned the degree of differences between them as a dendrogram (Fig. 2B). Accordingly, the Venn diagram was used to illustrate the number of unique and shared differentially-expressed gene for each cell type. The extent of unique differentially expressed genes for each cell type is as follows: $98.33 \%$ (59 of 60 ) for PNG, $100 \%$ (1 of 1) for SPG, $90 \%$ (36 of 40) for CG, 60\% (3 of 5) for EG, 78.95 (75 of 95) for ALG and 96.39 (454 of 471) for Neurons (Fig. 2C).

\section{Identification of deathstaras a gene with specific enrichment in astrocyte-like glia of $D$. melanogaster}

After cell type identification, we asked whether specific genes can also characterize the identified cell types. To identify the genes capable of distinguishing different cell types, we performed differential expression analysis in order to compare the transcriptome of the cluster of interest with that of all other cell types. The differentially expressed genes (DEGs) for each cell type are presented in Supplemental file S2.

Among all the clustered cells, ALG could be sufficiently discriminated by a single uncharacterized gene named CG11000 (here assigned as deathstar). Expression level analysis of this gene using the RNA-seq profile of single cells in Drosophila optic lobe [12] demonstrated its significant enrichment in 12 cells of all 84 cells, which corresponds to ALG (Fig. 3A). In the same RNA-seq expression data (accession number: GSE103771), a significant positive correlation $\left(R^{2}=0.6637\right.$; $p$-value $\left.=0.0001\right)$ was observed between the expression level of the candidate gene (deathstar) and that of Eaat1, which previously was reported as ALG-specific marker [5] (Fig. 3B). In addition, the expression pattern of deathstar and Eaat1 across developmental time of $D$. melanogaster, derived from a different RNA-seq data (accession number: GSE107049) showed a correlation between these two genes (Fig. 3C and 3D), supporting the observed expression specificity of deathstargene in ALG of D. melanogaster.

To corroborate the assigned specificity of deathstar, we tested whether the promoter-GAL4 lines of this gene could label ALG in CNS of D. melanogaster. Consistently, data showed that deathstar (Red signals) is mostly co-labelled with the ALG marker Eaat-1 (Green signals), in specific bilateral parts of Drosophila optic lobe and VNC. Both male and female flies showed the same pattern of deathstar expression in Drosophila CNS (Fig. 4A and 4B). However, the signals of deathstargene in two cells of Drosophila VNC were not overlapped with the expression of Eaat1 gene. This experiment was performed twice for males and females, and the same results were obtained (Supplemental file S3).

\section{Sex-biased developmental effect of deathstargene in D. melanogaster}


We crossed GAL4 strains that are expressed in each neural cell types of $D$. melanogaster with the deathstar-dsRNA to knockdown deathstar protein level in each neural cell types. Our results showed that most of the males expressing deathstar-RNAi in all neural cell types are lethal during development. However, the females with the same genotype developed normally to adult. Both males and females in the control group (harbouring deathstar-RNAi without GAL4 expression) showed normal development (Fig. 5A). This result suggests that the deathstar gene may have a developmental function exclusively in male flies. However, such developmental effect was not cell type-specific. As the control of this experiment, the Drosophila CG15765 gene was examined in the same procedure, and a standard ratio of males over females $(\sim 1)$ was obtained for all genotypes (Fig. 5B). The exact number of the progenies of the corresponding crosses are summarized in the supplemental file S4.

\section{Knockdown of deathstargene affects locomotion activity of D. melanogaster}

It has been previously reported that ALG cells that cover specific territories of Drosophila CNS have significant effects on locomotion behaviours [13]. To investigate the role of deathstar gene in locomotion behaviour in $D$. melanogaster, we knocked down the deathstar protein level using RNAi in different cell types of CNS. Then we performed the climbing assay. Results showed that the flies that specifically express deathstar RNAi in astrocytes show significant defects in climbing ability (Fig. 6A), suggesting the crucial role of this gene in this cell type. Interestingly, we found that the knockdown of deathstar protein does not affect the climbing activity in female flies (Fig. 6B). When we knocked down the deathstar in EGN and SPG, however, female flies showed severe defects in climbing activity (Fig. 6B). These data suggest that the expression of deathstar in ALG cells is crucial only in males, not females regarding the climbing activity.

To test whether the behavioural locomotive effect of deathstar gene is altered during the ageing of $D$. melanogaster, the climbing assay was performed on the flies with different ages. Results showed that the older flies expressing deathstar-RNAi in all neural cell types (three- and four-week-old flies) exhibited significant climbing deficiency (Fig. 6C). However, when we tested the same genotype of flies in the oneand two-week stages, the climbing defects appeared in ALG-specific manner (Fig. 6C). The climbing defects were not observed in the flies with neuron-specific suppression of deathstar gene, suggesting the climbing defects related to deathstar is glial-specific in old flies (Fig. 6C). Unlike the male flies, both young and old females did not exhibit any meaningful pattern of climbing defects (Fig. 6D). All these data suggest that the expression of deathstarin male ALG cells is crucial for climbing ability in the early adult stage.

\section{Astrocyte-specific suppression of deathstargene shortens lifespan in D. melanogaster}

To test the possible function of deathstargene in D. melanogaster lifespan, we knocked down deathstar in distinct CNS cell types (CG, PNG, ALG, EGN, EGT, SPG, Glia and Neurons) and measured their life span for 40 50 days. We observed a significant reduction in lifespan for the flies that had ALG-specific expression of deathstar RNAi, supporting the specific effect of the deathstargene in ALG on the life span of D. melanogaster (Figure 7A). However, in female flies such reducing effect of 
deathstar RNAi was observed in other cell types (EGN, EGT, PNG and neurons) at lower level than that of ALG. The ALG-specific effect of deathstarRNAi on lifespan was observed in both male and female flies (Fig. 7A and 7B), suggesting that the role of deathstar on the lifespan is not sexually dimorphic.

\section{Discussion}

The brain of $D$. melanogaster is a complex organ with a diverse set of cells serving analogous functions to those of human [7]. With differential expression of the genes, each cell in the brain can determine its own fate to be a particular cell type with specific functional characteristics [12]. Previous studies identified and reported the existence of several cell types in nervous system of $D$. melanogasterassigned as; perineural glia (PNG), subperineural glia (SPG), cortex glia (CG), neuropil ensheathing glia (EGN), tract ensheathing glia (EGT), astrocyte-like glia (ALG) and neurons (Neu) [6].

Some of Drosophila genes has been reported in previous studies to be specifically expressed in a particular cell type of CNS [6]. These cell type-specific markers were frequently used for structural and functional annotation of different circuits in the nervous system of $D$. melanogaster [6]. In this study, the transcriptome of 55,000 single cells within the optic lobe of $D$. melanogaster brain was analyzed to identify novel cell type-specific genes followed by functional analysis of the genes. The optic lobe occupies about two-third of whole D. melanogaster brain [14] with diverse set of cells including the abovementioned cell types [12].

To determine the type of the sequenced single cells, we used previously-reported specific marker genes and the cells with the highest expression level of a particular marker and lack of expression for other markers. Then we classified our bioinformatical analysis with a specific cell type (Fig. 1). In order to test the accuracy of this classification, the selected single cells were compared to each other based on their whole transcriptome (e.i. 17272 Drosophila genes). Results showed successful clustering of most single cells into their corresponding cell type (Fig. 2A). Only the predicted SPG cells could not be classified in a single cluster possibly because of expression heterogeneity of their marker genes (Mdr65, moody and Gli genes) or differences in transcriptome due to their functional specification or physical territory changes within CNS. SPG cells were shown to be equivalent to the vertebrate bloodbrain barrier (BBB). Since they may possess the hallmarks of a potent interface between Drosophila brain and environment, their transcriptome can be influenced by their positioning and environmental circumstance [15]. Therefore, each single SPG cell may have its profile and could not be categorized in a single cluster based on the transcriptome. Similarly, shreds of evidence showed that ensheathing glia (EG) cells could change their expression profile to be involved in the immune system, thereby their transcriptome will be different from their initial identity [16].

While two specific markers classified PNG cells, CG4797 and gem, (Fig. 1), they were categorized well in two separate clusters based on their transcriptome, here assigned as PNG- $\alpha$ and PNG- $\beta$ (Fig. 2). This data suggests a new subtype of PNG in D. melanogaster. However, further computational and experimental works need to be performed for confirmation of the suggested subtypes. 
The best clustering result was found for ALG. Among the six analyzed cells, five cells were clustered together, and the other one was very close to this cluster (Fig. 2). Through comparison of the transcriptome of the cells in the ALG cluster to the other single cells of Drosophila optic lobe, the deathstar gene was found to be differentially enriched in ALG (Fig. 3A). Therefore, we hypothesized that this gene could be a novel ALG-specific gene and could mediate specific functions of ALG cells within the central nervous system of $D$. melanogaster.

In order to test this hypothesis, its expression was compared with the previously-known marker for ALG (Eaat1 gene) using two different expression datasets (GSE103771 and GSE107049). These data showed a significant positive correlation between deathstar and Eaat1 genes which suggest their similar pattern of expression in single cells of the optic lobe (Fig. 3B) and also across different stages of Drosophila brain development (Fig. 3C and 3D). Given the ALG-specificity of deathstar gene based on the analyzed RNA sequencing data, we decided to determine its expression pattern in the central nervous system of $D$. melanogaster using fluorescent labelling of the deathstargene by RFP and the known ALG marker (Eaat1) by GFP, driven by GAL4 and LexA proteins, respectively. Results showed strong bilateral signals corresponding to six single cells for each side of ventral nerve cord and also many single cells within optic lobe (Fig. 4, red signals). However, while the expression of desthstar-GAL4 mostly covers ALG cells, some of GFP signals show broader expression pattern of this GAL4 driver. The insertion site of this GAL4 may affect the broader expression pattern. However, we showed that the previsously known ALGspecific cells can be labeled by deathstar-GAL4 driver. We will produce more specific GAL4 strains in future studies.

Such specific territories of ALG cells in the ventral nerve cord of $D$. melanogaster were also reported by Peco et al., who attributed some locomotion behaviours of $D$. melanogaster to these cells [13]. According to these findings, the locomotion behaviour of $D$. melanogaster was assessed under the suppression of the deathstargene in male and female flies. For this purpose, male flies expressing GAL4 in different neural cell types were crossed with virgin females harbouring the UAS-deathstar-RNAi (Table 1). Unexpectedly, we found that the number of newly-eclosed male progenies was drastically lower than females. The collection of male and female flies was repeated for six times, and the same result was obtained (Supplemental file S4). After counting and quantification of the data, we found that while the progenies of the control group (Canton-S $\times$ UAS-deathstarRNAi) shows the same ratio of male and female flies, all other progenies expressing deathstar RNAi shows the female-biased ratio of progenies (Fig. 5A). For the control of the experiment, another Drosophila gene named CG15765 was analyzed in the same procedure parallel with the deathstar gene. Results of CG15765 gene showed the same ratio for males and females in all conditions (Fig. 5B). Suppression of the deathstar gene in female flies did not show any developmental defect or visible phenotype change (Fig. 5A). These data suggest that the deathstargene is specifically involved in the development of male $D$. melanogaster.

Interestingly, the survived male flies showed a reduced locomotion activity, exclusively when the deathstargene was suppressed in ALG cell type, supporting the specificity of this gene in ALG cells in adulthood stages of $D$. melanogaster. However, deathstarsuppression in females did not show any 
significant changes in climbing activity (Fig. 6). Females showed an EGN- and SPG-specific reduction of climbing activity. However, this effect was not observed throughout their lifespan (Fig. 6B and 6D). These results suggest that while the expression pattern of the deathstargene is similar in both sexes of $D$. melanogaster (Fig. 4), it has differential effects on locomotion behaviours in male and female flies.

There are other pieces of evidence for ALG-enriched gene that have different patterns of expression during early and late stages of $D$. melanogaster development. ALG-enriched genes in larval stages are mainly involved in metabolism, energy production, and protein synthesis, consistent with the known role of astrocytes in metabolic support of neurons [17]. In comparison with the larval astrocytes, ALG cells in adult $D$. melanogaster acquire a new function for regulation of behaviors, additional to their functions in earlier stages [17].

Therefore, it is expected that the suppression of the deathstargene in larval stages is lethal in males. However, this suppression at adulthood stages mediates climbing defects in males (Fig. 5 and 6).

Analysis of lifespan in the flies expressing deathstar RNAi in different cell types showed an ALG-specific reduction of lifespan in males which could be another supportive evidence for specificity of deathstargene to ALG cell type (Fig. 7). It is known that Drosophila astrocytes (ALG) are significant contributors to modulate neurotransmitter homeostasis within synapses [18] and also regulators of circadian rhythm [19].

Therefore, it is likely that abolishing the functions of ALG or ALG-specific genes could interfere with normal locomotion properties and lifespan of D. melanogaster, which are the direct outcome of dysregulations in neurotransmission and circadian rhythm, respectively. Moreover, it was recently reported that mitochondrial dysfunctions in ALG cells contribute to neurodegeneration $[18,20]$, which could give rise to shortened lifespan of D. melanogaster[21].

\section{Conclusions}

Taken together, deathstar (previously known as CG11000) was reported as a protein-coding gene in a fruit fly with 332 amino acids. Its functional role was not known yet in Drosophila, and it has no homologue gene in human (according to Flybase reports). In this study, we firstly annotated deathstaras an ALGspecific gene which could mediate the developmental process of the male flies additional to its functions in locomotion and lifespan properties of $D$. melanogaster. Further experiments need to be performed in order to elucidate the molecular mechanisms underlying the observed developmental and behavioural effects of this gene in $D$. melanogaster.

\section{Materials And Methods}

\section{Dataset}


A single-cell RNA-sequencing (RNA-seq) data of a recently published paper [12] was selected from the data repository NCBI Gene Expression Omnibus (GEO) (accession number: GSE103771). It contained the expression profile of 17272 Drosophila genes in a population of 55000 single cells from the optic lobe of Drosophila brain. Also, the data of expression profile for the whole brain of $D$. melanogaster across four different developmental stages (days 5, 10, 20 and 40) were downloaded from NCBI GEO (accession number: GSE107049).

\section{Bioinformatics analyses}

For expression analysis of the genes in single cells of $D$. melanogaster brain, the expression matrix belonging to the study of Konstantinides et al. [12] was downloaded for downstream analyses. We analyzed the expression matrix of the single cell RNA-seq data of Drosophila optic lobe (accession number: GSE103771) based on the expression level of known markers previously reported for each cell type. Based on the expression pattern of the specific markers, the type of the cells in the matrix was characterized. For each cell type, six single cells were selected according to the expression level of the markers. In total, forty two cells comprising seven cell types of Drosophila nervous system (assigned as perineural glia (PNG), subperineural glia (SPG), cortex glia (CG), neuropil ensheathing glia (EGN), tract ensheathing glia (EGT), astrocyte-like glia (ALG) and neurons (Neu)) were selected for downstream analyses. For confirmation of this cell type classification, hierarchical clustering was performed for the selected forty-two cells based on their similarities in transcriptome and presented as a heatmap with colour codes for the Pearson correlation coefficient. The clusters with at least three cells (of all six cells) were validated as unique cell type. To identify the genes enriched in a specific cell type, we first identified differentially expressed genes (DEGs) between the examined cell types and all other cells by the R package limma [22]. Then, after applying adjusted $P$-values (Benjamini-Hochberg correction) of $<0.05$ and $\log _{2} \mathrm{FC} \geq 1.5$, the specificity of the candidate genes with highest degree of enrichment was determined by analysis of their expression across all the examined single cells (unpaired t-test; $P$-value $<0.05$ ). Venn diagram was obtained from the web-based tool on http://bioinformatics.psb.ugent.be/webtools/Venn/. 


\section{Fly stocks and crosses}

All the Drosophila melanogaster lines in this study were prepared from Bloomington Drosophila Stock Center (BDSC). After receiving the stocks they were cultured in standard conditions (standard cornmeal-agar yeast fly food at $25^{\circ} \mathrm{C}$ on a 12-h light:12-h dark condition) as previously described [23]. $\mathrm{CO}_{2}$ was used as an anesthetic. For functional analysis of the genes, the GAL4/UAS-RNAi system [24] was used to deplete their functions in a cell-type specific manner. The fly stocks together with their crosses for functional analyses of deathstar gene are summarized in table 1. After crossing the virgin females of UAS-deathstar-RNAi line with males of different GAL4 driver lines (x-GAL4), ensuring expression of RNAi in cell type-specific manner, the transgenic progenies (x-GAL4 > UASRNAi) were collected based on their genotypes and assessed for their behaviors (Table 1). The specific GAL4 driver gene for each cell type was as below: CG4797 gene for PNG, Mdr65 gene for SPG, Cyp4g15 gene for CG, CG9657 gene for EGN, CG34340 gene for EGT, Eaat1 gene for ALG, repo gene for all glial cells (glia) and $n S y b$ gene for neurons. For control of the experiment flies without expression of RNAi were used. These flies had dsRNAi in their genome without expression of GAL4. Therefore no functional RNAi was produced in the control flies. All of our experiments were performed in Canton-S background to rule out all the genetic background effects.

For the crosses of fluorescent labeling experiment, the two binary systems of GAL4/UASGFP and LexA/LexAoP-RFP were used simultaneously in an individual male and female flies. The ALG cells of Drosophila CNS were labelled by the expression of a membranebound GFP ( $\mathrm{mCD}_{8} \mathrm{GFP}$ ) under the control of Eaat1 gene promoter, fused to the sequence of LexA (Eaat1-LexA). For tracing the expression pattern of deathstar gene in Drosophila CNS, a transgenic fly line carrying deathstar-GAL4 construct (BDSC\#: 23418) was used. Expression of RFP under the control of deathstar gene promoter depicts the expression distribution of deathstar gene across CNS. The crossing scheme for this experiment is summarized in table 2. 


\begin{tabular}{|c|c|c|}
\hline \multicolumn{2}{|c|}{ Parents for crosses } & \multirow[t]{2}{*}{ Progenies } \\
\hline UAS- RNAi lines ( $(\square)$ & GAL4 lines ( $(\square)$ & \\
\hline $\begin{array}{l}\mathrm{w}^{-} ;+/+ \text {; deathstar-RNAi } \\
(\# \text { 51918) }\end{array}$ & $+;+/+;+/+$ & - \\
\hline $\mathrm{w}^{-} ;+/+$; deathstar-RNAi & + ; +/+ ; PNG-GAL4/ TM3 & Non-TM3 \\
\hline $\mathrm{w}^{-} ;+/+$; deathstar-RNAi & + ; +/+ ; SPG-GAL4/ TM3 & Non-TM3 \\
\hline $\mathrm{w}^{-} ;+/+$; deathstar-RNAi & $+;+/+$; CG-GAL4/ TM3 & Non-TM3 \\
\hline $\mathrm{w}^{-} ;+/+$; deathstar-RNAi & + ; +/+ ; EGN-GAL4/ TM3 & Non-TM3 \\
\hline $\mathrm{w}^{-} ;+/+$; deathstar-RNAi & + ; +/+ ; EGT-GAL4/ TM3 & Non-TM3 \\
\hline $\mathrm{w}^{-} ;+/+$; deathstar-RNAi & + ; +/+ ; ALG-GAL4/ TM6B & Non-TM6B \\
\hline $\mathrm{w}^{-} ;+/+$; deathstar-RNAi & $+;+/+$; Neuron-GAL4/ TM6B & Non-TM6B \\
\hline
\end{tabular}

Table 1. Summary of crosses and experimental genotypes for behavioral analyses. In order to generate the transgenic flies with expression of deathstar-RNAi in cell type-specific manner, the male flies of different GAL4 driver lines were crossed with the females harboring UAS-deathstar-dsRNAi in their genome (BDSC\#: 51918). All the females ( $\square$ ) for these crosses were virgin. Among the progenies of these crosses, the flies with proper genotypes were selected based on the absence of balancer chromosomes (CyO, TM3 and TM6B).

\begin{tabular}{|l|l|l|}
\hline Lines & Fly stocks (Genotypes and Bloomington ID) & Cross scheme \\
\hline A & $\begin{array}{l}\text { UAS-RFP, lexAop-GFP ; Sp/CyO ; TM2/TM6B } \\
\text { (\# 32229) }\end{array}$ & \\
\hline B & W- ; ALG-LexA/CyO ; TM2/TM6B & \\
\hline C & UAS-RFP, lexAop-GFP ; ALG-LexA/CyO ; TM2/TM6B & \\
\hline D & W- ; +/+ ; deathstar -GAL4 (\# 23416) & \\
\hline E & UAS-RFP, lexAop-mCD8GFP ; ALG-LexA/+ ; TM6B/ deathstar -GAL4 & \\
\hline
\end{tabular}

Table 2. Crossing scheme for imaging experiments of CNS in Drosophila. The line A contains RFP and GFP reporter genes under regulation of UAS and LexAop sequences, respectively. Line B expresses LexA protein in an ALG-specific manner. By crossing the lines A and B (Parents), the fly line $\mathrm{C}$ ( $\mathrm{F}_{1}$ generation) was produced and then crossed with deathstar -GAL4 line (D) to produce $F_{2}$ generation. Among the flies of $F_{2}$, the males and 
females that lack balancer chromosomes but contain the components of the binary systems UAS/GAL4 and LexA/LexAop were selected for central nervous system (CNS) dissection. The females ( $\square$ ) of all crosses were virgin.

\section{Immunostaining and imaging}

As described before [25], brains dissected from adults 5 days after eclosion were fixed in $4 \%$ formaldehyde for $30 \mathrm{~min}$ at room temperature, washed with $1 \%$ PBT three times (30 min each) and blocked in 5\% normal donkey serum for 30 min. The brains were then incubated with primary anti-bodies in $1 \% \mathrm{PBT}$ at $4^{\circ} \mathrm{C}$ overnight followed with fluorophoreconjugated secondary antibodies for 1 hour at room temperature. Brains were mounted with antifade mounting solution (Invitrogen, catalog \#S2828) on slides for imaging.

Primary antibodies used here are chicken anti-GFP (Aves Labs, 1:1000), rabbit anti-DsRed express (Clontech, 1:250) and mouse anti-Bruchpilot (nc82) (DSHB, 1:50). Fluorophoreconjugated secondary antibodies used here are Alexa Fluor 488-conjugated goat antichicken (Invitrogen, 1:100). Alexa Fluor 488-conjugated donkey anti-rabbit (Invitrogen, 1:100), RRX-conjugated donkey anti-rabbit (Jackson Laboratory, 1:100), and Dylight 405conjugated donkey anti-mouse (Jackson Laboratory, 1:100).

Images were collected using a Zeiss LSM 7 MP microscope scanning confocal microscope. Projection of the confocal stacks, adjusting the brightness and contrast of the images were performed by NIH ImageJ [26], then presented by Adobe Illustrator CC 2019 for windows.

\section{Developmental assays}

To assess the possible function of deathstar gene in the development of $D$. melanogaster from pupae to adulthood stage, the newly eclosed D. melanogaster adults of the crosses in table 1, were collected and counted based on their genotypes. After counting, data were presented as the percentage of male and female flies with deathstar RNAi to the total number of eclosed flies. 


\section{Lifespan assay}

Lifespan was measured at room temperature according to standard protocols. In brief, newly ecloded flies ( 0 to 3 days) were collected (50 per genotype) and then placed in vials (25 flies per vial) and transferred to fresh vials every three days. Survival was recorded for each genotype (two vials). We scored flies stacked in the food as death events in all the vials analyzed. We created survival curves with Prism version 6 (GraphPad Software, San Diego, CA, USA) using the method of Kaplan and Meier. For statistical significance of the experiment, one-way ANOVA method was used and the death rate of each genotype was compared to the Canton-S (CS) group as the control. ${ }^{*} P$-value $<0.05,{ }^{* *} P$-value $<0.01$.

\section{Climbing assay}

For the climbing assay, flies were separated into groups of 25 adults per each vial and kept in $29^{\circ} \mathrm{C}$. At the time of experiment, the groups of 25 flies were placed in an empty climbing vial and then tapped down to the bottom. They were allowed to climb from the bottom to top of the vial $(8 \mathrm{~cm})$ for 20 seconds. In order to evaluate the climbing pattern of each genotype, the climbing height achieved by each genotype was calculated in every 5 seconds after the tapping and initiating negative geotaxis. The results of climbing assay were obtained by video recording of climbing flies then processed by ImageJ software. Data was presented as graphs using Prism version 6 (GraphPad Software, San Diego, CA, USA). Flies with Canton-S genotype were used as control group.

\section{Statistical analysis}

Statistical analysis and graphic representations were performed using Prism 6.00 for Windows (GraphPad Software, San Diego, CA, USA). Unpaired t test or one-way ANOVA (using the Bonferroni's multiple comparison) were applied depending on the measurements analyzed in the corresponding experiment. In all cases averages with SEM are plotted. $P$ values were calculated by two-tailed unpaired Student's t-test unless otherwise specified, using GraphPad Prism 6 software. All data are presented as the mean \pm SEM. $* P<0.05$; $* * P<0.01 ;$ and $* * * P<0.001$. 


\section{List Of Abbreviations}

ALG: Astrocyte-like glia; BBB: Blood-brain barrier; CG: Cortex glia; CNS: Central nervous system; CS:

Canton S; DEG: Differentially-expressed gene; dsRNA: double strand RNA; EGN: Neuropil ensheathing glia; EGT: Tract ensheathing glia; GEO: Gene expression Omnibus; NEU: Neurons; PNG: Perineural glia; RNAi: RNA interference; SPG: Subperineural glia; GFP: Green fluorescent protein; RFP: Green fluorescent protein.

\section{Declarations}

Ethics approval and consent to participate: Not applicable.

Consent for publication: Not applicable.

Availability of data and materials: Not applicable.

\section{Competing interests:}

The authors declare that they have no competing interests.

\section{Funding:}

No external fund was received for this study.

\section{Author contributions:}

Concept and design of experiments: WJK, $\mathrm{HN}$ and KW; performing the experiments: $\mathrm{HN}, \mathrm{KW}$ and WJK; acquisition of data: $\mathrm{HN}, \mathrm{KW}$ and WJK; analysis and interpretation of data: $\mathrm{HN}$, KW and WJK; drafting or revising the article: $\mathrm{HN}$ and WJK.

\section{Acknowledgments:}

We thank the Bloomington Stock Center for flies. We also thank all laboratory members for their kind supportive helps and advices during performing the study, especially Ammar Salkini and Khoi-Nguyen Ha Nguyen for their contribution to experiments performed in this manuscript.

\section{References}

1. Zeng Z, Miao N, Sun T. Revealing cellular and molecular complexity of the central nervous system using single cell sequencing. Stem cell research \& therapy. 2018;9(1):234.

2. Brody T, Odenwald WF. Cellular diversity in the developing nervous system: a temporal view from Drosophila. Development. 2002;129(16):3763-70.

3. Olesnicky E, Wright E. Drosophila as a model for assessing the function of RNA-binding proteins during neurogenesis and neurological disease. Journal of developmental biology. 2018;6(3):21. 
4. Tolwinski N. Introduction: Drosophila-A Model System for Developmental Biology. Multidisciplinary Digital Publishing Institute; 2017.

5. Yildirim K, Petri J, Kottmeier R, Klämbt C. Drosophila glia: Few cell types and many conserved functions. Glia. 2019;67(1):5-26.

6. Kremer MC, Jung C, Batelli S, Rubin GM, Gaul U. The glia of the adult D rosophila nervous system. Glia. 2017;65(4):606-38.

7. Freeman MR. Drosophila central nervous system glia. Cold Spring Harbor perspectives in biology. 2015;7(11):a020552.

8. Shih M-FM, Davis FP, Henry GL, Dubnau J. Nuclear transcriptomes of the seven neuronal cell types that constitute the Drosophila mushroom bodies. G3: Genes, Genomes, Genetics. 2019;9(1):81-94.

9. Croset V, Treiber CD, Waddell S. Cellular diversity in the Drosophila midbrain revealed by single-cell transcriptomics. Elife. 2018;7:e34550.

10. Henry GL, Davis FP, Picard S, Eddy SR. Cell type-specific genomics of Drosophila neurons. Nucleic acids research. 2012;40(19):9691-704.

11. Hilu-Dadia R, Hakim-Mishnaevski K, Levy-Adam F, Kurant E. Draper-mediated JNK signaling is required for glial phagocytosis of apoptotic neurons during Drosophila metamorphosis. Glia. 2018;66(7):1520-32.

12. Konstantinides N, Kapuralin K, Fadil C, Barboza L, Satija R, Desplan C. Phenotypic convergence: distinct transcription factors regulate common terminal features. Cell. 2018;174(3):622-35. e13.

13. Peco E, Davla S, Camp D, Stacey SM, Landgraf M, van Meyel DJ. Drosophila astrocytes cover specific territories of the CNS neuropil and are instructed to differentiate by Prospero, a key effector of Notch. Development. 2016;143(7):1170-81.

14. Tix S, Minden JS, Technau G. Pre-existing neuronal pathways in the developing optic lobes of Drosophila. Development. 1989;105(4):739-46.

15. DeSalvo MK, Hindle SJ, Rusan ZM, Orng S, Eddison M, Halliwill K, et al. The Drosophila surface glia transcriptome: evolutionary conserved blood-brain barrier processes. Frontiers in neuroscience. 2014;8:346.

16. Doherty J, Logan MA, Taşdemir ÖE, Freeman MR. Ensheathing glia function as phagocytes in the adult Drosophila brain. Journal of Neuroscience. 2009;29(15):4768-81.

17. Huang Y, Ng FS, Jackson FR. Comparison of larval and adult Drosophila astrocytes reveals stagespecific gene expression profiles. G3: Genes, Genomes, Genetics. 2015;5(4):551-8.

18. Logan MA. Glial contributions to neuronal health and disease: new insights from Drosophila. Curr Opin Neurobiol. 2017;47:162-7.

19. Ng FS, Tangredi MM, Jackson FR. Glial cells physiologically modulate clock neurons and circadian behavior in a calcium-dependent manner. Curr Biol. 2011;21(8):625-34.

20. Liu L, Zhang K, Sandoval H, Yamamoto S, Jaiswal M, Sanz E, et al. Glial lipid droplets and ROS induced by mitochondrial defects promote neurodegeneration. Cell. 2015;160(1-2):177-90. 
21. Kounatidis I, Chtarbanova S. Role of glial immunity in lifespan determination: a Drosophila perspective. Frontiers in immunology. 2018;9:1362.

22. Ritchie ME, Phipson B, Wu D, Hu Y, Law CW, Shi W, et al. limma powers differential expression analyses for RNA-sequencing and microarray studies. Nucleic acids research. 2015;43(7):e47-e.

23. Guo A, Li L, Xia S, Feng C, Wolf R, Heisenberg M. Conditioned visual flight orientation in Drosophila: dependence on age, practice, and diet. Learning \& Memory. 1996;3(1):49-59.

24. Brand AH, Perrimon N. Targeted gene expression as a means of altering cell fates and generating dominant phenotypes. development. 1993;118(2):401-15.

25. Kim WJ, Jan LY, Jan YN. A PDF/NPF neuropeptide signaling circuitry of male Drosophila melanogaster controls rival-induced prolonged mating. Neuron. 2013;80(5):1190-205.

26. Schneider CA, Rasband WS, Eliceiri KW. NIH Image to ImageJ: 25 years of image analysis. Nature methods. 2012;9(7):671.

\section{Supplemental Data Captions}

Supplemental file S1. Hierarchical clustering of single cells selected based on specific markers for neural cell types of $D$. melanogaster. Among all cell types, the ALG and neurons had the best clustering pattern.

Supplemental file S2. List of differentially expressed genes for each cell type of Drosophila central nervous system. The genes with $\log _{2}$ fold change $\left(\log _{2} \mathrm{FC}\right) \geq 1.5$ and $P$-value $<0.05$ were considered as differentially expressed genes (Denoted as highlighted cells).

Supplemental file S3. Expression pattern analysis of deathstargene in central nervous system of $D$. melanogaster by immunostaining. Many cells of optic lobe and several single cells within the ventral nerve cord were labeled by the RFP that was tagged to deathstar promoter (arrowheads). Many of the labeled cells by deathstar were overlapped with the signals of the known ALG marker, Eaat1 gene, also shown by arrowheads in the merged photos. Top and bottom panels are immunostaining of CNS in male

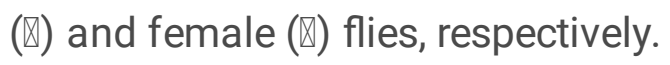

Supplemental file S4. Number of newly eclosed flies under suppression of deathstargene in neural cell types of $D$. melanogaster. The number of male progenies was less than the females when the deathstar expression was suppressed in neural cell types of $D$. melanogaster (RNAi carriers). Balancer carriers refer to the progenies which had no dsRNAi in their genome. As control of the experiment, a fly line which had no expression of deathstarRNAi. Equal number of males and females was observed for this control (CS) group.

\section{Figures}


B

\begin{tabular}{|c|c|}
\hline Cell type & Marker gene \\
\hline \multirow{2}{*}{ PNG } & CG4797 \\
\cline { 2 - 2 } & gem \\
\hline \multirow{2}{*}{ SPG } & Mdr65 \\
\cline { 2 - 2 } & moody \\
\cline { 2 - 2 } & Gli \\
\hline CG & Cyp4g15 \\
\hline EGN & CG9657 \\
\hline \multirow{2}{*}{ EGT } & CG34340 \\
\hline ALG & Eaat1 \\
\hline \multirow{2}{*}{ NEU } & nSyb \\
\cline { 2 - 2 } & elav \\
\hline
\end{tabular}
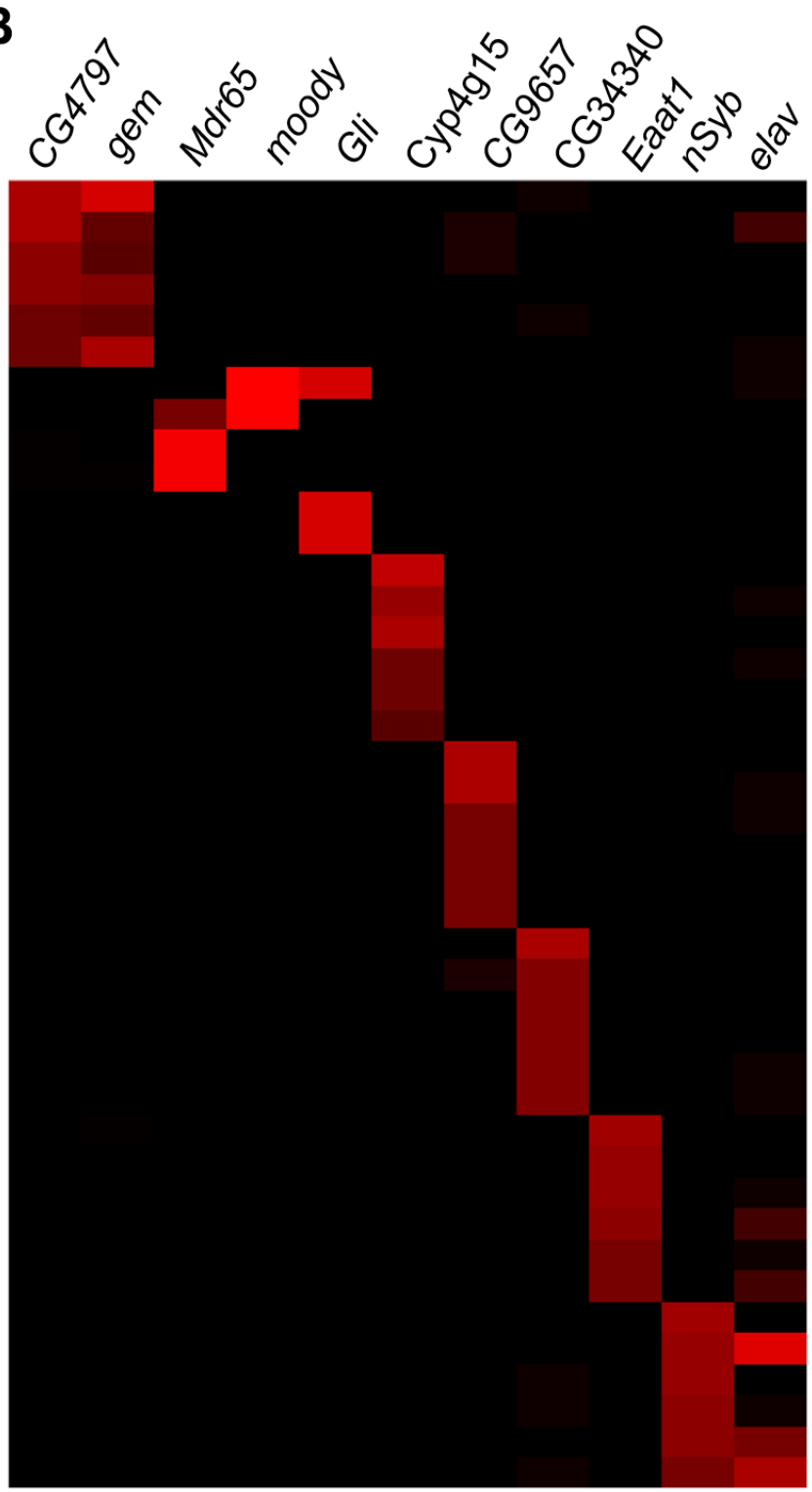

Cell 156

Cell_533

Cell_870

Cell_1013

Cell_662

Cell_1239

Cell_2

Cell_12658

Cell_12741

Cell_25003

Cell_8701

Cell 31749

Cell_2296

Cell_726

Cell_318

Cell 5174

Cell_6311

Cell_3603

Cell_46711

Cell 51046

Cell_756

Cell_57266

Cell_27194

Cell_13107

Cell 62168

Cell 1556

Cell 61161

Cell_42740

Cell 39257

Cell_10924

Cell 2569

Cell_19

Cell_127

Cell_502

Cell 143

Cell_423

Cell 41

Cell_24

Cell 22

Cell_38

Cell_36

Cell_44

\section{Figure 1}

Identification of Drosophila neural cell types through analysis of their specific marker genes. A) Specific marker genes, previously reported as markers for each cell type of Drosophila glial cells. B) Heatmap of gene expression for 42 selected cells from 55000 single sequenced cells, in which, six different cells were identified as a unique cell type (denoted by different colors). Cells' numbers correspond to the numbering method of Konstantinides et al. Red and black colors indicate the marker-positive and marker-negative cells, respectively. Color intensities in the heatmap denote the level of gene expression based on RPKM per single cell. 
A

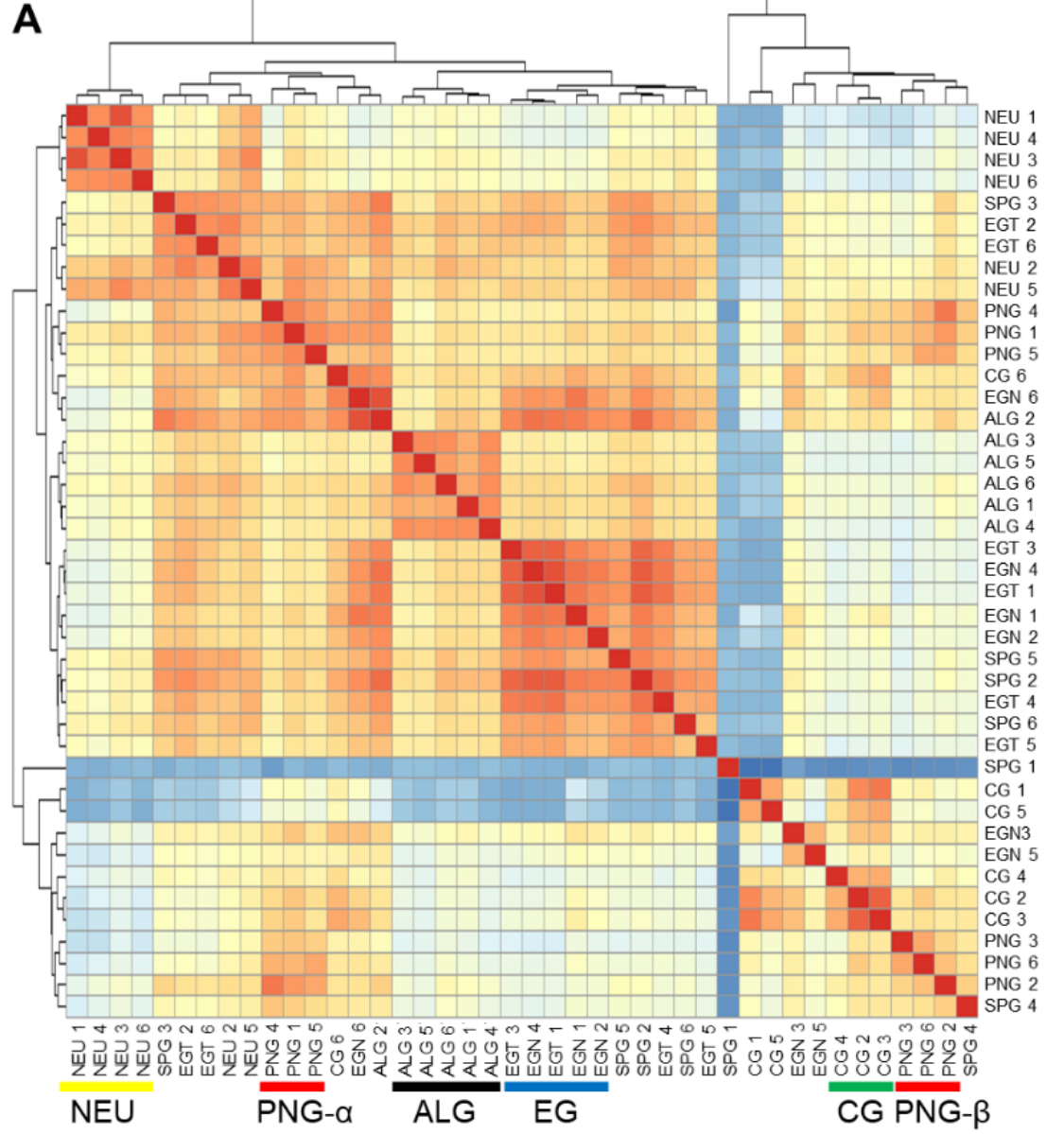

B
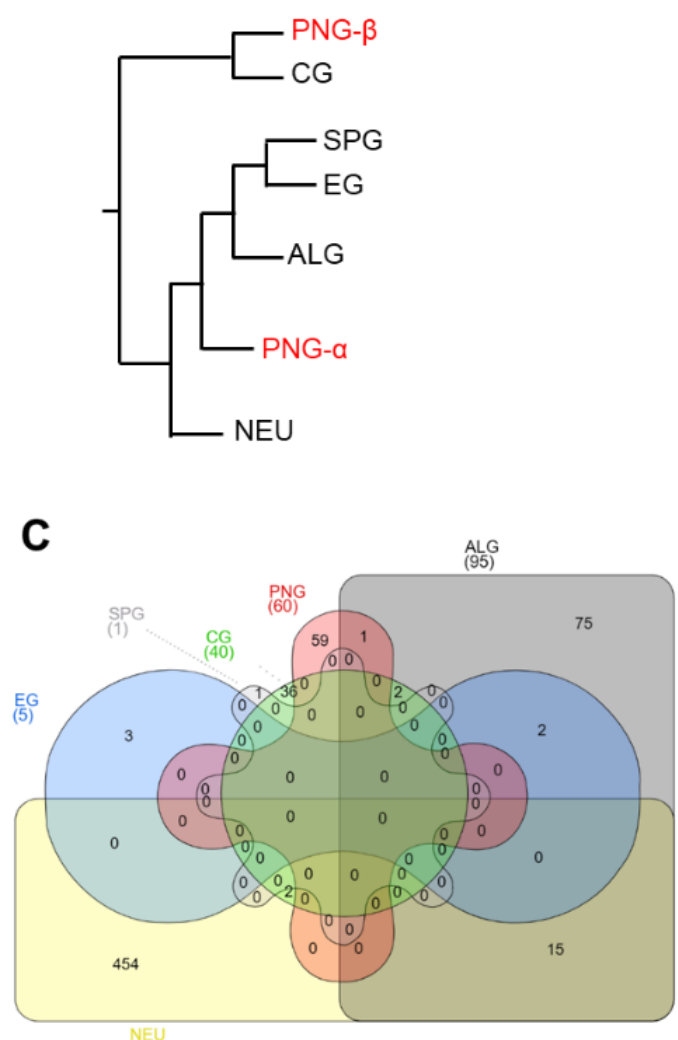

Figure 2

Hierarchical clustering of different neural cell types of D. melanogaster based on their transcriptome. A) Hierarchical cluster analysis of gene expression for 42 selected cells from Drosophila optic lobe based on the expression level of 17,272 genes for each single cell. Except SPG, all the studied cell types were clustered together suggesting their similarities in expression profile. PNG cells were strongly clustered in two different clusters, suggesting presence of two distinct PNG subtypes in Drosophila (PNG-a and PNG$\beta)$ B) Dendrogram shows the similarity degree of the clustered cells based on whole transcriptome. C) Number of unique and shared DEGs for each cell type of D. melanogaster nervous system. Total number of DEGs for each cell type are presented in parenthesis. $P N G=$ perineural glia, SPG= subperineural glia, $\mathrm{CG}=$ cortex glia, $\mathrm{ALG}=$ astrocyte-like glia, $\mathrm{EG}=$ ensheathing glia, and $\mathrm{NEU}=$ neurons . 

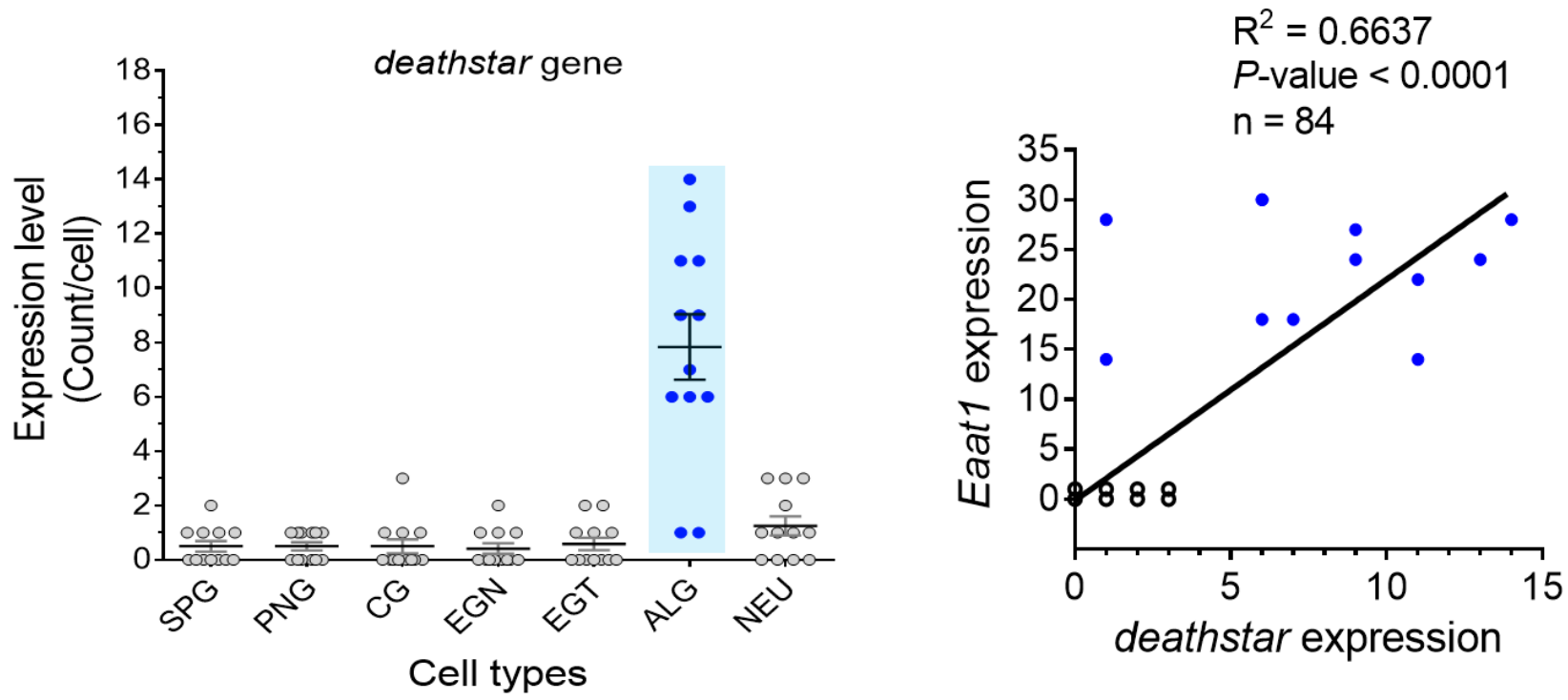

C

Male

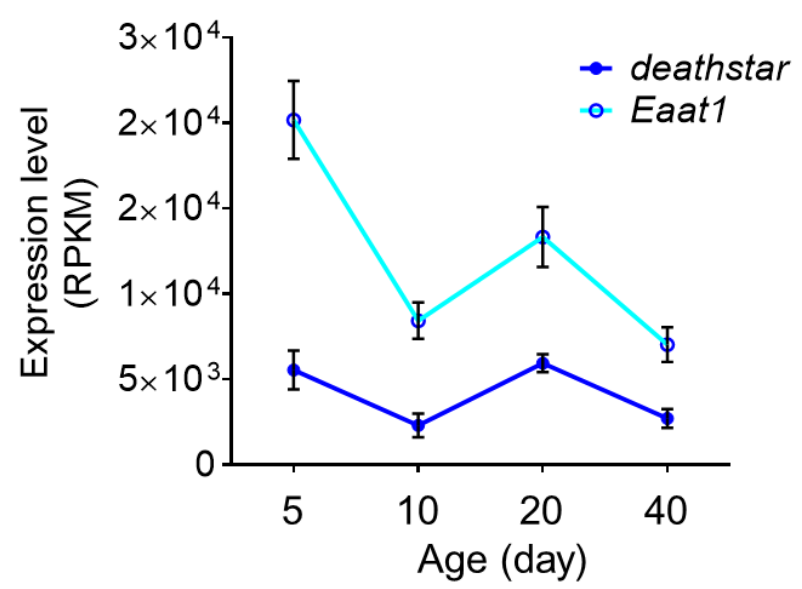

D

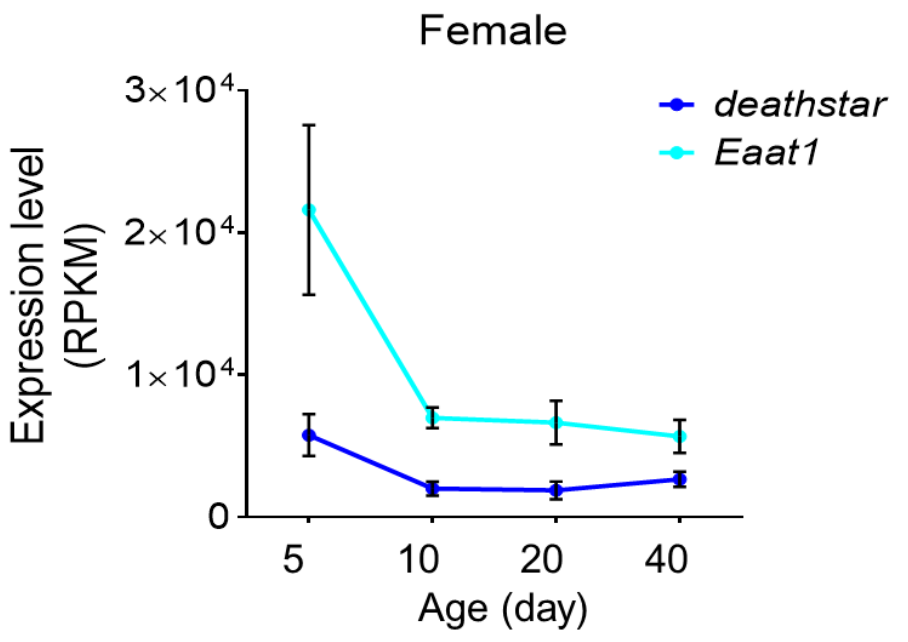

Figure 3

Expression specificity of deathstar gene in ALG cells of D. melanogaster. A) While the ALG cells exhibited high level of deathstar mRNA (Blue points), other glia cells as well as neurons showed low or no expression of this gene (gray points). B) Positive correlation between expression level of known ALG marker (Eaat1 gene) and that of deathstar gene. Blue and gray points respectively denote expression of the genes in ALG and non-ALG single cells. C) Similar expression pattern of deathstar (dark blue) and Eaat1 (bright blue) genes during development of male D. melanogaster. D) Similar expression pattern of deathstar (dark blue) and Eaat1 (bright blue) genes during development of female D. melanogaster. Developmental time is presented as the days after eclosion of the flies. SPG= subperineural glia, PNG= 
perineural glia, $\mathrm{CG}=$ cortex glia, $\mathrm{ALG}=$ astrocyte-like glia, EGN= neuropil ensheathing glia, EGT= tract ensheathing glia, and NEU= neurons.

A

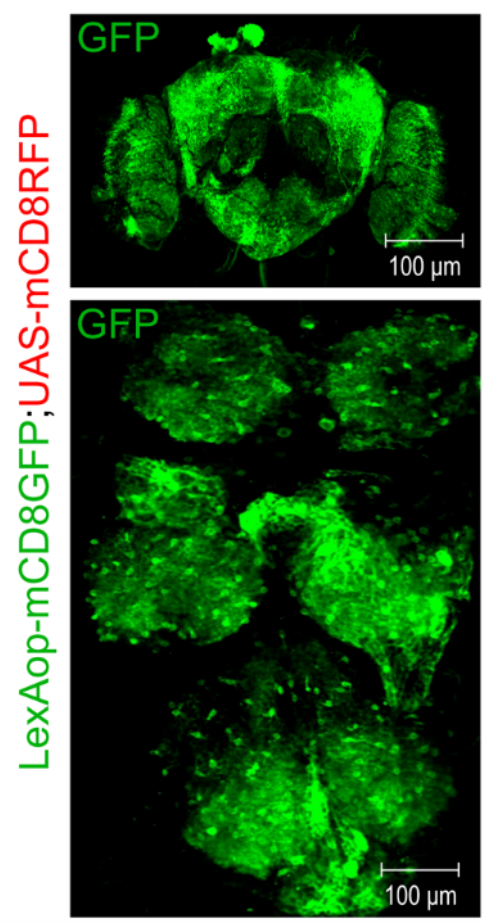

B

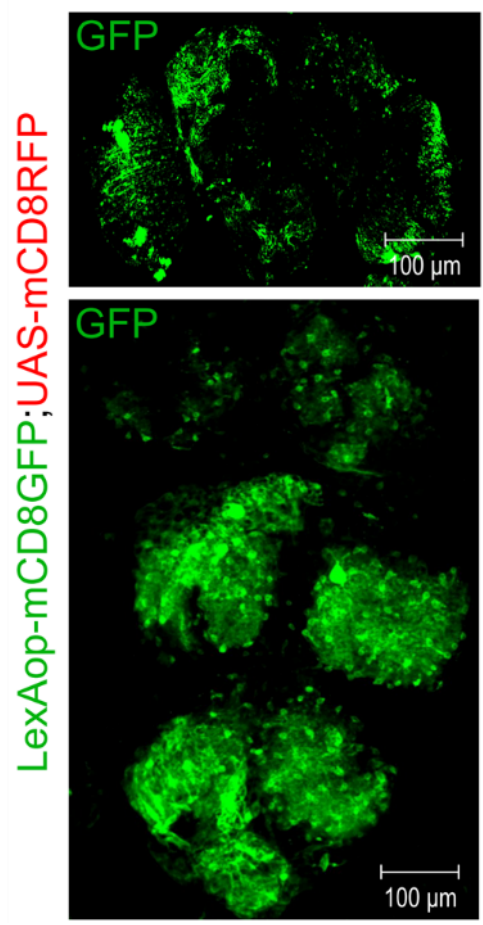

Eaat1-LexA;deathstar-GAL4
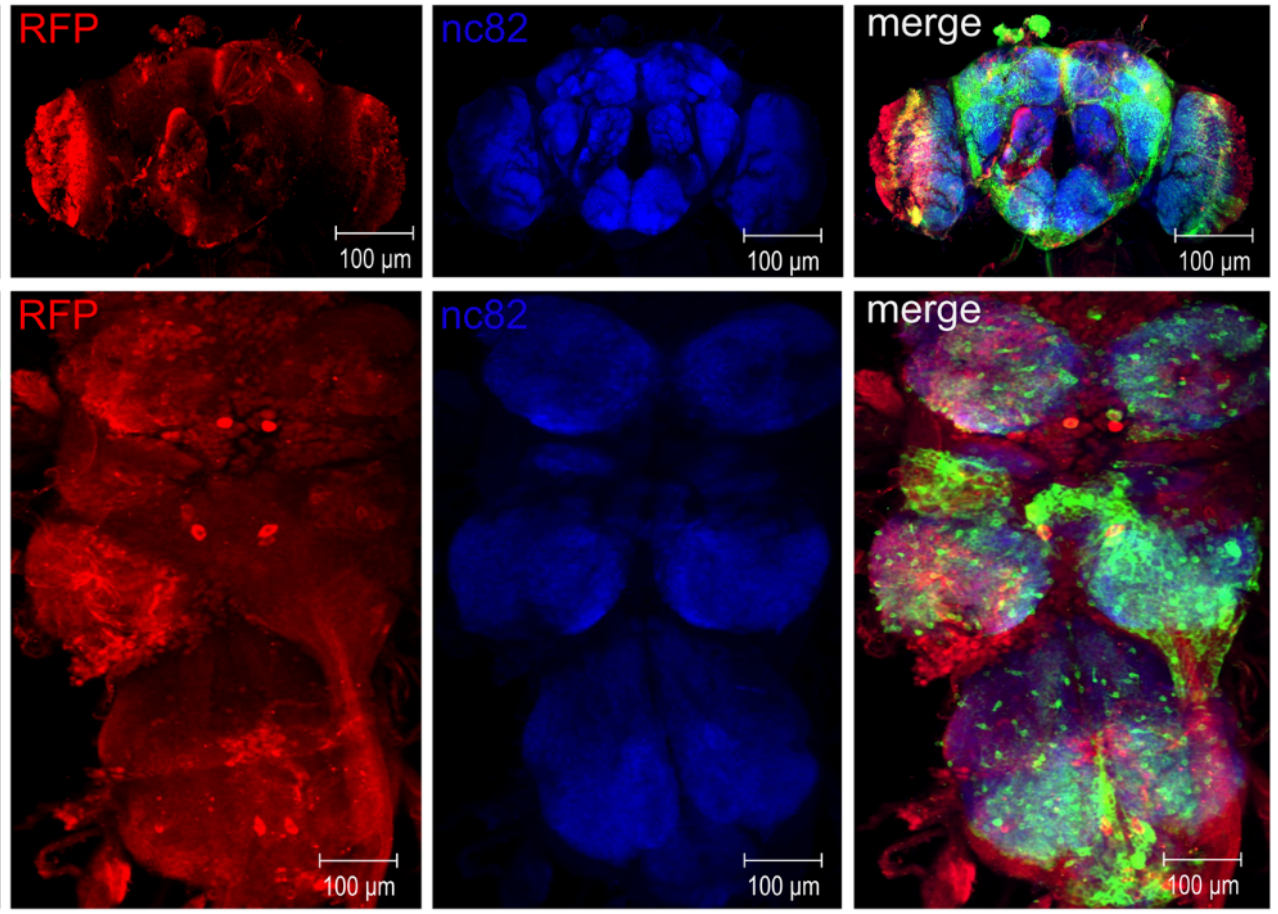

Eaat1-LexA;deathstar-GAL4
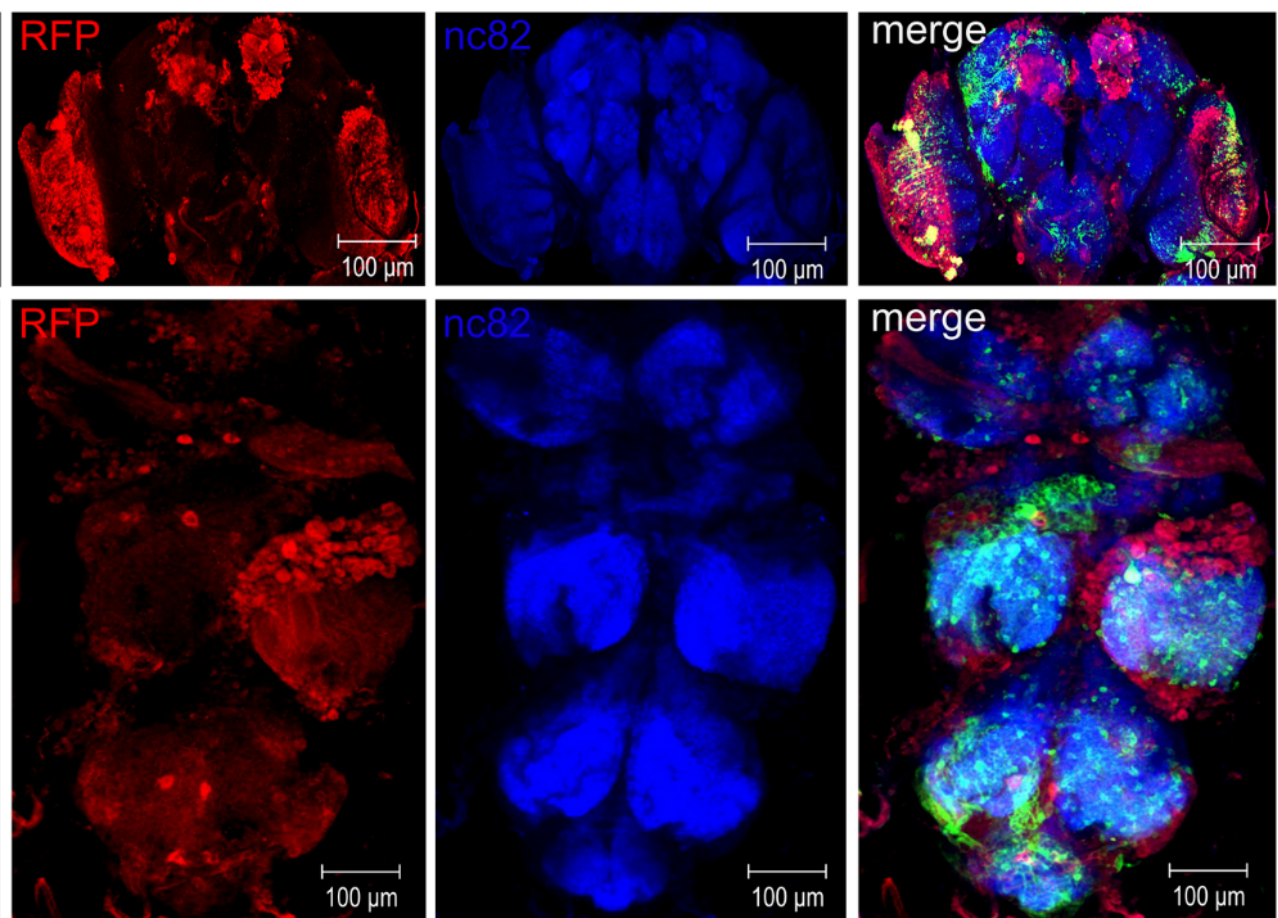

\section{Figure 4}

deathstar gene is differentially expressed in Drosophila ALG cells. Immunostaining result of CNS in male (A) and female (B) flies. In both images, the ALG cells were stained with anti-GFP (green), and the Red signals (anti-RFP) reflect UAS-RFP driven by a GAL4, tagged by the promoter region of deathstar gene 
(deathstar-GAL4). Anti-nc82 was used to label neuropil (Blue). Co-labeling of astrocytic cells using Eaat1LexA/LexAop-mCD8GFP (green) together with deathstar-GAL4/UAS-RFP (Red) showed ALG-specific expression of deathstar gene among other types of Drosophila glia cells. Scale bars represent $100 \mu \mathrm{m}$ in all image parts.

A

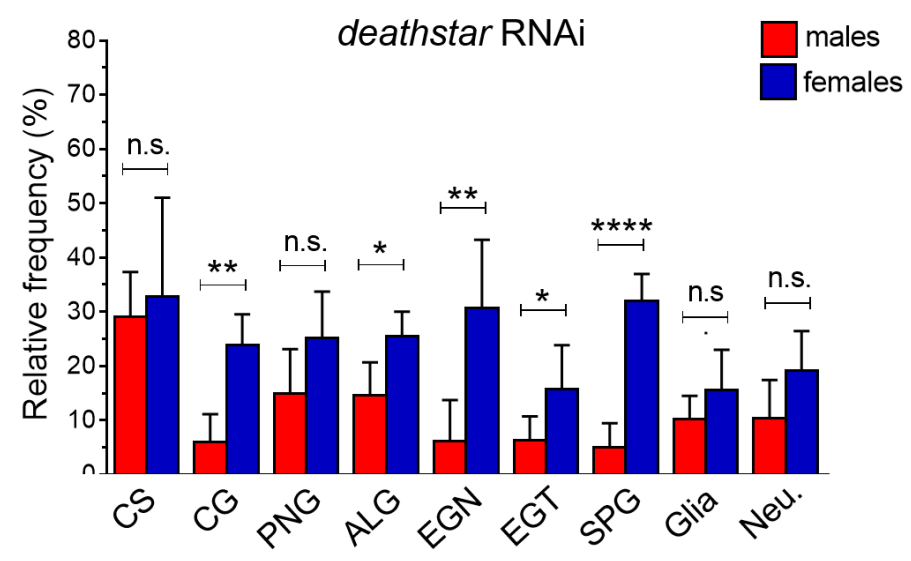

B

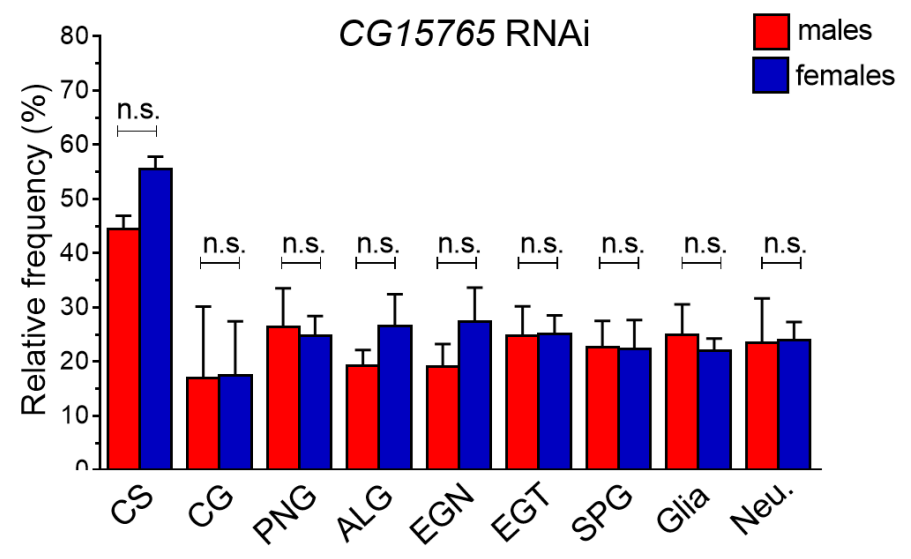

Figure 5

Male-specific developmental effect of deathstar gene in D. melanogaster. A) While the females with deathstar RNAi showed normal development, male flies with the same genotypes could not be matured to adults and showed overall less frequencies in comparison with the expected ratio of each genotype. B) Using another RNAi as control (CG15765 RNAi), equal number of males and females were observed which were consistent with the expected ratio of each genotype. $* \mathrm{P}<0.05 ; * * \mathrm{P}<0.01 ; * * * \mathrm{P}<0.001$ and $* * * * \mathrm{P}<0.0001$, n.s. $=$ non-significant $(\mathrm{P} \geq 0.05)$. 
A

deathstar RNAi in males

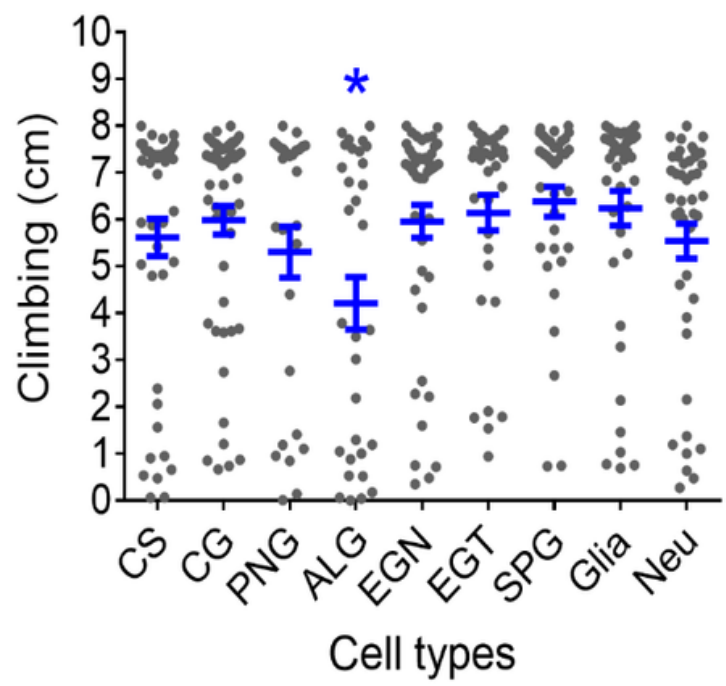

C

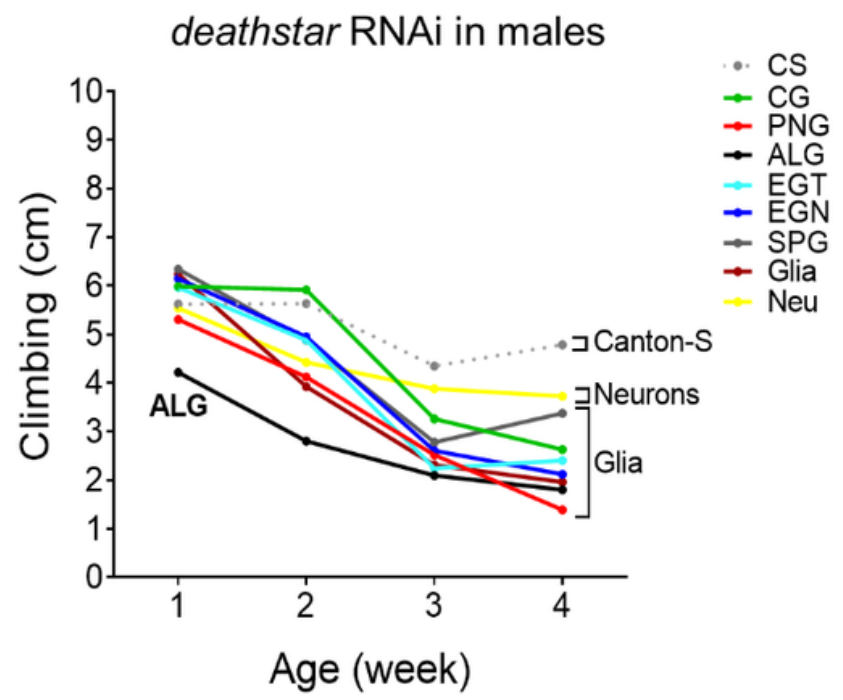

B

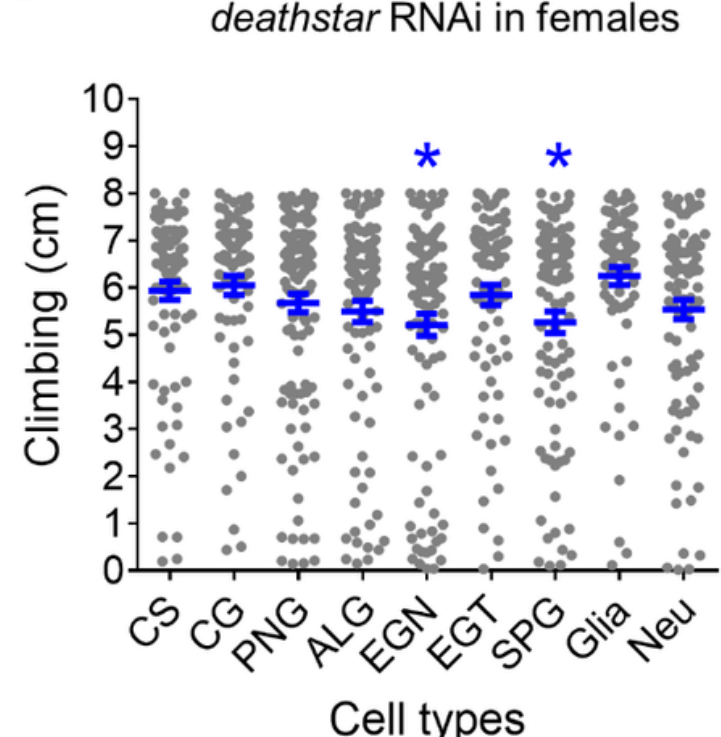

D

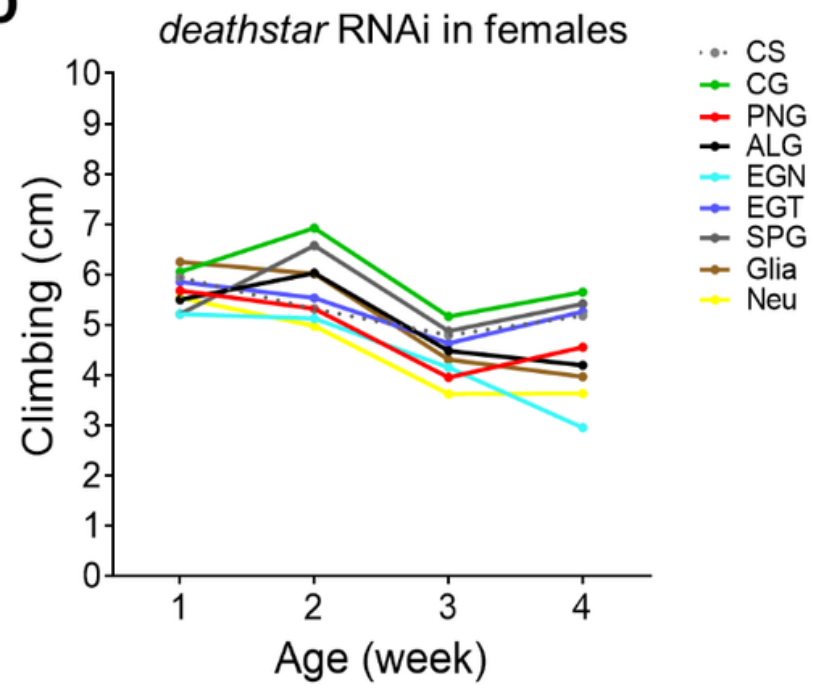

Figure 6

Effects of deathstar gene suppression on locomotion behavior of D. melanogaster. A) ALG-specific reduction of climbing activity in male flies under suppression of deathstar gene. B) Slight EGN- and SPGspecific reduction of climbing behavior in female $D$. melanogaster under suppression of deathstar gene. C) Climbing behaviors of male D. melanogaster expressing deathstar RNAi during different times of development (weeks 1-4) showing ALG-specific effect of the gene. D) Climbing behaviors of female D. melanogaster expressing deathstar RNAi during different times of development (weeks 1-4) showed no significant effect in ageing flies. 


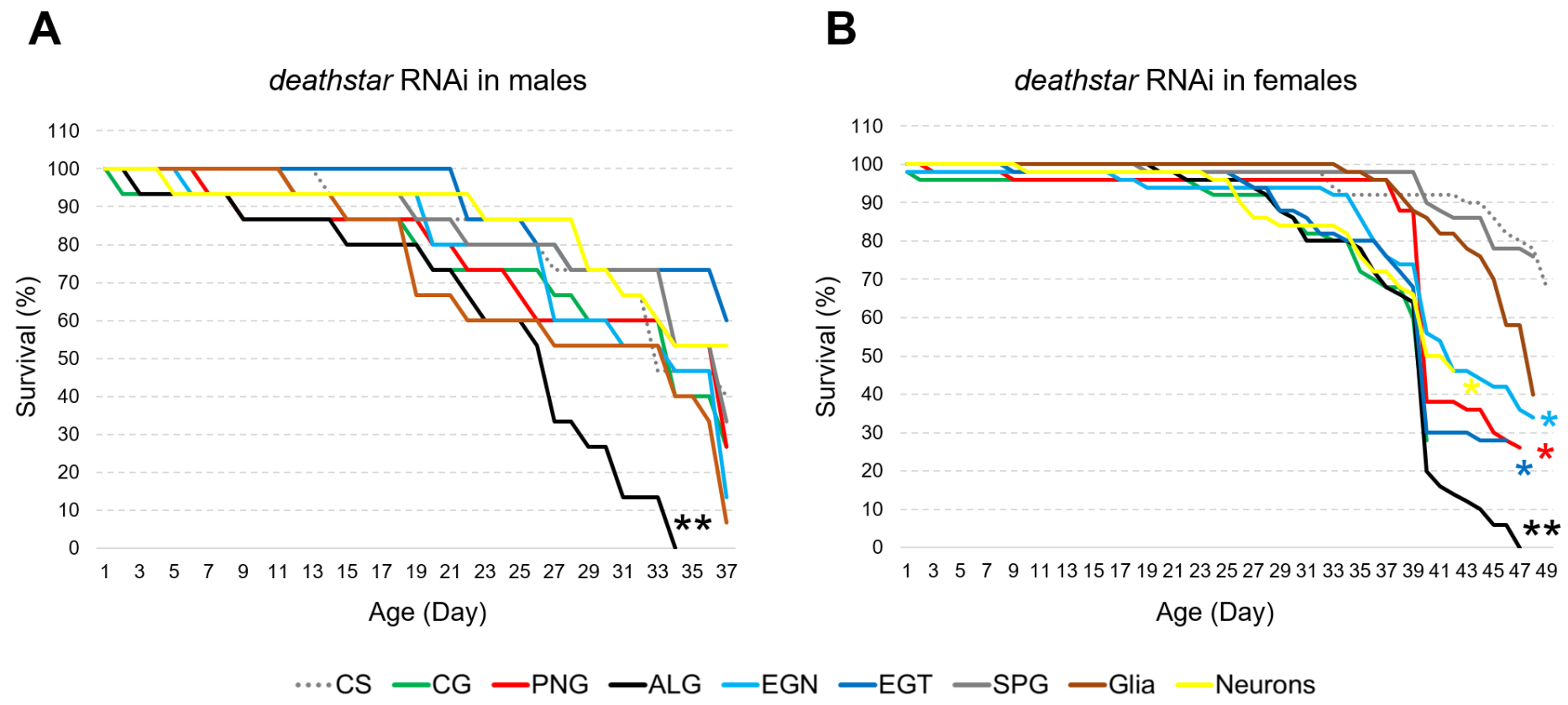

\section{Figure 7}

Lifespan effect of deathstar gene suppression in D. melanogaster. Suppression of deathstar gene shortened lifespan of male and female flies in an ALG-specific manner. Only the males with ALG-specific suppression of deathstar showed significant reduction in survival. In females the greatest level of lifespan reduction was observed in the flies with ALG-specific suppression of deathstar. However, females carrying deathstar RNAi in their EGN, EGT, PNG and neurons showed such an effect.

\section{Supplementary Files}

This is a list of supplementary files associated with this preprint. Click to download.

- SupplementalFileS3.pdf

- Supplementalfiles2.xlsx

- Supplementalfiles4.pdf

- SupplementalFileS1.pdf 